\title{
Überall und nirgendwo - \\ „Disability Mainstreaming“ im kommunalen Lebensraum und Sozialraumorientierung als Transformationskonzept
}

\author{
Elisabeth Wacker
}

I. Nirgendwo - die (Un-)Sichtbarkeit von Behinderung und der Lebenschancenansatz

II. Sozialraum und ICF - Ansatzpunkte zur Transformation eines Lebensraums für alle

III. Überall - „Mainstreaming Disability“ als Entwicklungsprogramm und -methode

IV. Ausblick - evidenzbasierte Maßstäbe für Leistungssysteme als Königsweg?

V. Literatur

Die Veränderung kommunaler Praktiken im Umgang mit Personen mit Beeinträchtigung und Benachteiligung lohnt grundlegend neuer sozialwissenschaftlicher Aufmerksamkeit, auch wenn oder gerade weil geradlinige Prozesse des Wandels schwer aufzuzeigen sind. Vielmehr liegen - wie der Wissenschaftshistoriker Rheinberger ${ }^{1}$ dies beschreibt - wie in einem Bergwerk Goldadern nicht offen, sondern müssen entdeckt werden. Zwar stehen Wege in Form bereits gegrabener Tunnel und Schächte den Suchenden zur Verfügung, diese sind aber teilweise verwirrend angeordnet und Sicherheit, zielführend unterwegs zu sein, ist nicht einfach zu erlangen. Deswegen mag ein multidisziplinärer Blick zunächst als Verwirrung erscheinen, aber er kann auch als VerheiBung gedeutet werden, der Gefahr eindimensionaler Pfadfindung aus spezifisch fachlicher Perspektive zu entrinnen.

Wenn die Frage lautet, wie im Sozialraum Neues entsteht nach der Maßgabe einer aktuellen Vision der inklusiven Gesellschaft, so kann es verschiedene fachliche Tunnel und methodische Schächte geben, um dem Kern des Wissens näher zu kommen, das im Licht der Behindertenrechtskonvention der Vereinten Nationen (UN-BRK) ${ }^{2}$ neu zu betrachten ist. Der hier abgesteckte „Claim“ bezieht sich auf einen noch utopischen Le-

1 Rheinberger, Man weiß nicht genau, was man nicht weiß. Über die Kunst, das Unbekannte zu erforschen, NZZ vom 5.5.2007.

2 Das Übereinkommen der Vereinten Nationen vom 13. Dezember 2006 über die Rechte von Menschen mit Behinderung sowie das Fakultativprotokoll zum Übereinkommen sind in Deutschland seit 2009 geltendes Recht in Form einfachen Bundesrechts (BGBl. II 2008, S. 1419 ff.). 
bensraum „Kommune“, in dem Merkmale und Möglichkeiten von Transformationskonzepten in drei Gedankensträngen aufgezeigt werden sollen: erstens die Sichtbarkeit von Behinderung durch einen Lebenschancenansatz, zweitens der Wandel von Sozialräumen zum Lebensraum aller und drittens die Potenziale eines „Disability Mainstreaming“ als Katalysator kommunaler Entwicklung 3 .

\section{Nirgendwo - die (Un-)Sichtbarkeit von Behinderung und der Lebenschancenansatz}

Es gibt einige Bevölkerungsgruppen wie „die Alten“, „die Arbeitslosen“ oder „die Alleinerziehenden“, deren gesellschaftliche Verankerung fragil erscheint. Aber „die Behinderten“ befinden sich in Gefahr, traditionell und von Anfang an zu den „Ausgeschlossenen“" zu zählen.

Das Risiko einer Kombination von Ungleichheit und Benachteiligung tritt bei physisch, psychisch oder sinnesbeeinträchtigten Menschen charakteristisch auf. Dies kann mehrere Gründe haben:

- es ist deutsche Tradition, dass Maßnahmen und Aktivitäten von Politik und Leistungsträgern dann einsetzen, wenn Behinderung eingetreten ist;

- noch immer wird Behinderung vorrangig so betrachtet, wie es Prüfdaten medizinisch-therapeutischer Diagnostiken oder leistungsbezogene Verwaltungsstatistiken nahelegen (z.B. schädigungsorientiert bzw. nach Grad der Behinderung: $\mathrm{GdB})$;

- Leistungen erfolgen vorrangig über den Einsatz „besonderer Maßnahmen“ (bzw. in Sondereinrichtungen).

Daher wird die Exklusion dieses Personenkreises über Strukturen und Organisationen verfestigt ${ }^{4}$, die wiederum die Inklusionschancen am Arbeitsmarkt, in privaten und öffentlichen Organisationen, in allen Stufen des Bildungssystems (von der Vorschule bis zur Universität), bei politischer Willensbildung und -gestaltung, aber auch im Bürgerschaftlichen Engagement sowie in der alltäglichen Lebensführung beeinflussen. Dies führt in einen ,Teufelskreis“.

Die tatsächlichen Lebensbedingungen der behinderten Menschen sind hingegen kaum im Blickfeld, hier fehlt es also

- an gesellschaftlicher Wahrnehmung und Problembewusstsein,

- an wissenschaftlich fundierten Kenntnissen zur Lebenslage von Menschen mit Beeinträchtigungen, um besser zu wissen und zu verstehen, inwieweit räumliche,

3 Grüber, Disability Mainstreaming, IMEW konkret Nr. 10, Dezember 2007, abrufbar unter: www. imew.de/index.php?id=237 (letzter Zugriff: 19.03.2011).

4 Bude/Lantermann, Soziale Exklusion und Exklusionsempfinden, Kölner Zeitschrift für Soziologie und Sozialpsychologie 58/2006, S. 233-252. 
soziale und Bedingungen der Infrastruktur gleichberechtigte Teilhabe bei Beeinträchtigung behindern sowie

- an Forschung zu Potenzialen sowie ihrem Aufbau bzw. ihrer Stärkung, damit es den sehr verschieden beeinträchtigten Menschen gleichermaßen möglich wird, selbstbestimmt und ohne Teilhabeeinschränkungen zu leben.

Eine Ungleichheit scheint allerdings in rechtlicher Perspektive zunächst kaum kenntlich, denn die Maßgabe: „Niemand darf wegen seiner Behinderung benachteiligt werden" ist seit nunmehr beinahe zwei Jahrzehnten in Artikel 3 des Grundgesetzes (GG5) aufgenommen. Dieses Diskriminierungsverbot enthält allerdings paradoxe Züge, folgt man einer sozialwissenschaftlichen Definition von Behinderung, die genau in der Benachteiligung der Beeinträchtigten ihren Ausgangspunkt nimmt (d.h. Behinderung bedeutet eben keine Wesenheit oder Eigenschaft einer Person, sondern die Benachteiligung der Personen mit dem Merkmal einer oder mehrerer Beeinträchtigungen). Hier könnte mit der Aufnahme eines Verbots der „Benachteiligung von Behinderten“ ins Grundgesetz sachlogisch also ein Pyrrhussieg errungen sein, gerade im Sinne der Gleichstellung beeinträchtigter Menschen. Denn die Abweichung durch Beeinträchtigung (des Sehens, Gehens, Entscheidens, Orientierens oder anderer Funktionalitäten) wird scheinbar unverrückbar mit Behinderung gekoppelt.

Gesellschaftliche Realität wird in einer aktuellen Umfrage der Aktion Mensch erkennbar, die belegt, dass über die Hälfte der Bevölkerung in Deutschland behinderten Menschen im Alltag nicht begegnet. Zwar wünschen sich demnach 87 \% der Deutschen ein Zusammenleben auf gleicher Augenhöhe, aber jede/r Dritte gibt an, gar keinen Kontakt zu Menschen mit Beeinträchtigung zu haben6. Die Exklusion scheint also Normalität zu sein, obwohl seit dem Jahr 2001 ein eigenes Sozialgesetzbuch (SGB IX7) Rehabilitation und Teilhabe als sozialpolitische Zielorientierung ausweist.

Die mit der UN-BRK (s.u. III.) von Deutschland unterzeichnete Zielsetzung gesellschaftlicher Inklusion bei Beeinträchtigung könnte daher als aus menschenrechtlicher Perspektive getriebene Vision interpretiert werden, auf die es vor allem hinzuarbeiten gilt. Die Existenz von Behinderung alltagsnah in einer inklusiven Gesellschaft aufzuheben, wäre dann schrittweise zu entwickeln, indem

- nach einem Lebenschancenansatz Beeinträchtigung und Benachteiligung in ihrem Zusammenwirken identifiziert und sichtbar werden und zugleich

- präventive, rehabilitative und soziale Leistungen bezogen auf ihre Wirkung beim Ausgleich von Benachteiligung geprüft werden, nach dem Maßstab der Teilhabegerechtigkeit.

5 Grundgesetz für die Bundesrepublik Deutschland (1949/Stand: 2012) BGBl. I 1994, S. 3146.

6 www.aktion-mensch.de, zit. nach Rehabilitation 2012; S. 51, 132.

7 Sozialgesetzbuch Neuntes Buch - Rehabilitation und Teilhabe behinderter Menschen, BGB1. I 2001, S. 1046. 
Der Lebenschancenansatz überschreitet traditionelle Zuordnungen zu sozialen Bezugskonzepten, wie Klasse, Schicht oder Milieu, zugunsten einer Diversitätsperspektive mit Aufmerksamkeit für verschiedene Lebenslagen und Ungleichheitsmerkmale in ihnen, wie Alter, Bildung, Ethnie, Funktionalität/Gesundheit/Körper, Geschlecht, Nationalität, Sexualität, Religion etc. ${ }^{8}$. In einem prozessorientiert angelegten Zugang kann reflektiert werden, ob es sich lohnt, Charakteristika des Lebens mit Behinderung in der Gesellschaft (bzw. die Verfügbarkeit von Ressourcen bei Beeinträchtigungen) entlang verschiedener Merkmale (Differenzlinien) zu verdeutlichen und zugleich nach deren verstärkenden, aufhebenden oder mildernden Wirkzusammenhängen zu fragen, wie dies beispielsweise die Intersektionalitätsforschung fordert und entwickelt ${ }^{9}$. Dieser mehrdimensionale Zugang kann zugleich den Druck mildern, sich bei einer vorliegenden Benachteiligung zugunsten eines Leistungsbezugs einer bestimmten Gruppe zuzuordnen bzw. ihrer Interessenvertretung zu unterwerfen.

Das Allgemeine Gleichbehandlungsgesetz $\left(\mathrm{AGG}^{10}\right)$ aus dem Jahre 2006 soll Benachteiligung verhindern oder beseitigen beim Zugang zum Arbeitsleben, zur Gesundheitsversorgung, Bildung oder anderen Gemeinschaftsgütern. Es nennt mehrere Merkmale, anhand derer Ungleichbehandlung und Benachteiligung sichtbar werden kann: In $\S 1$ „Ziel des Gesetzes“ werden Rasse oder ethnische Herkunft, Geschlecht, Religion oder Weltanschauung, Behinderung, Alter oder sexuelle Identität aufgezählt als mögliche Auslöser von Benachteiligung. Unterschieden wird weiter zwischen

- unmittelbarer Benachteiligung $\S 3$ Abs. 1 im Fall einer weniger günstigen Behandlung einer Person in vergleichbaren Situationen,

- mittelbarer Benachteiligung § 3 Abs. 2 im Fall scheinbar neutraler Vorschriften, die Personen mit den genannten Merkmalen benachteiligen,

- Belästigung bzw. sexueller Belästigung § 3 Abs. 3 und 4 als Benachteiligung, die die Würde einer Person und ihre sexuelle Integrität verletzt und der

- Anweisung zur Benachteiligung § 3 Abs. 5.

8 Sen, Ökonomie für den Menschen, München 2007; Voges/Jürgens/Mauer/Meyer, Methoden und Grundlagen des Lebenslagenansatzes, ZeS: Zentrum für Sozialpolitik Endbericht, 2003, www. soziologie.uni-kiel.de/bergersozun/Voges_Lebenslagenansatz.pdf.

9 Vgl. Baer/Bittner/Göttschke, Mehrdimensionale Diskriminierung - Begriffe, Theorien und juristische Analyse. Teilexpertise im Auftrag der Antidiskrimimierungsstelle des Bundes, Berlin 2011; Dern/Inowlocki/Oberlies, Mehrdimensionale Diskriminierung - Eine empirische Untersuchung anhand von autobiographisch-narrativen Interviews. Teilexpertise im Auftrag der Antidiskriminierungsstelle des Bundes, Berlin 2011; Lutz/Wenning, Differenzen über Differenz - Einführung in die Debatten, in: dies. (Hrsg.), Unterschiedlich verschieden. Differenz in der Erziehungswissenschaft, Opladen 2001, S. 11-24; Minow, Making All the Difference. Inclusion, Exclusion and American Law, Ithaka, New York 1990; Young, Justice and the Politics of Difference, Princeton, New Jersey 1990; Zinsmeister, Mehrdimensionale Diskriminierung. Das Recht behinderter Frauen auf Gleichberechtigung und seine Gewährleistung durch Art. 3 GG und das einfache Recht, Baden-Baden 2007.

10 Allgemeines Gleichbehandlungsgesetz, BGBl. I 2006, S. 1897. 
Auf der Basis des AGG wird es möglich, auch mehrere Diskriminierungsgründe gemeinsam zu berücksichtigen ( $(4$ : „Unterschiedliche Behandlung wegen mehrerer Gründe").

Aus der Perspektive eines Lebenschancenansatzes lässt sich Behinderung in der Gesellschaft folglich sichtbar machen ${ }^{11}$, indem

- die generelle Rolle und Position beeinträchtigter Menschen in der jeweiligen Gesellschaft thematisiert wird,

- Differenzen deutlich werden zur allgemeinen Lebenslage und

- soziale Leistungen für diesen Personenkreis und ihre Wirkungen und Nebenwirkungen auf den Prüfstand gestellt werden können.

Merkmale sozialer Beteiligung bzw. Benachteiligung im Arbeitsleben, am Wohnungsmarkt, bei der Mobilität, der Information und Kommunikation etc. werden benennbar.

Entsprechende Fragen können systematisch der Struktur gesellschaftlicher Binnendifferenzierung folgen und prüfen, wie der Zugang zu Gütern wie

- Waren und Dienstleistungen,

- politischem System,

- Bildungssystem,

- kulturellem Leben und

- Gesundheitssystem,

um nur einige Beispiele zu nennen, konkret behindert bzw. möglich wird.

Wirkungen von Maßnahmen können als Reduktion von Exklusion im Sinne unfreiwilligen Ausschlusses evident und ihre Auslöser damit erkennbar werden.

Dieser Zugang ist national ebenso wie transnational fruchtbar und kann bei systematischer Umsetzung ein Monitoring jeweiliger nationaler und binnenstruktureller (kommunaler) Entwicklungen ebenso ermöglichen wie die internationalen Kooperationen und Vergleiche, die die UN-BRK einfordert (Art. 31 ff.).

Über Bezugsgrößen des Lebenschancenansatzes werden neben Aussagen zur Lebenslage auch Ausprägungen von Inklusion und Exklusion identifizierbar, die soziale Anerkennung (Toleranz, Akzeptanz, Respekt ${ }^{12}$ ) und Zugehörigkeit verschieden beeinträchtigter Menschen in einer Gesellschaft über Beobachtung und Befragung (Selbstund Fremdauskünfte) abbilden.

Ziel dieser Strategie ist, Behinderung in der Gesellschaft sichtbar zu machen und so den Weg zur sozialen Inklusion zu ebnen, der vom Rat der Europäischen Union als Prozess beschrieben wird:

11 Wacker, Behinderung in der Gesellschaft. 50 Jahre im soziologischen Blick - vom Dialog zum Diskurs, Geistige Behinderung 47/2008, S. 42-61.

12 Sennett, Respekt im Zeitalter der Ungleichheit, Berlin 2004. 
„Social inclusion is a process which ensures that those at risk of poverty and social exclusion gain the opportunities and resources necessary to participate fully in economic, social and cultural life and to enjoy a standard of living and well-being that is considered normal in the society in which they live. It ensures that they have greater participation in decision making which affects their lives and access to their fundamental rights." 13

Auf welche Weise sich diese definierten Rechte auf Chancen und Ressourcen zur vollen Teilhabe und auf angemessene Standards von Lebensqualität und Wohlergehen realisieren, kann nicht alleine dem sozialpolitischen Spiel der Kräfte überlassen bleiben, sondern verpflichtet ebenso die Wissenschaft (insbesondere auch eine an Grundlagen orientierte Wissenschaft), die Frage zum Gegenstand zu machen.

Wie kann diese im Lebenschancenansatz operationalisierbare Aufgabe nun konkret werden?

\section{Sozialraum und ICF - Ansatzpunkte zur Transformation eines Lebensraums für alle}

Seit Jahren befassen sich insbesondere Sozialarbeit und Sozialpädagogik in ihren Analysen und Erprobungen mit dem Sozialraum ${ }^{14}$. An ihre Arbeiten kann angeknüpft werden. Sie betrachten den Sozialraum nicht einfach als einen Ort, sondern als Geschehensfeld, in dem das von Menschen konstruierte und immer aufs Neue rekonstruierte Leben Tag für Tag erkennbar wird. Dieser Alltagsraum ist angereichert mit Gefühlen, Möglichkeiten und Hindernissen. Hier realisiert sich regelgesteuertes und lebensweltliches Zusammenleben von Menschen. Lokalisiert im Dazwischen von Gesellschaft und Individuum ${ }^{15}$ richtet sich im Sozialraum Aufmerksamkeit auf die Wechselwirkungen zwischen sozialen Konstruktionen und Handelnden sowie auf deren Verflechtung. Insofern müssen auch Fragen, die sich auf soziale Wirklichkeit beziehen, auf Menschen in Situationen der Behinderung und Behinderungserfahrungen, im Kontext des Sozialrau-

13 Council of the European Union, Joint report by the Commission and the Council of social inclusion, SOC 115 ECOFIN 80 EDUC 46 SAN 49, Brüssel 2004, S. 10.

14 Bingel, Sozialraumorientierung revisited. Geschichte, Funktion und Theorie sozialraumbezogener Sozialer Arbeit, Wiesbaden 2011; Früchtel/Budde/Cyprian, Sozialer Raum und Soziale Arbeit. Textbook: Theoretische Grundlagen, Wiesbaden 2007; Hinte, Eigensinn und Lebensraum - zum Stand der Diskussion um das Fachkonzept „Sozialraumorientierung“, VHN 78/2009, 20-33; Kessl/Reutlinger, Sozialraum, in: Otto/Thiersch (Hrsg.), Handbuch Soziale Arbeit, 4. Aufl. München \& Basel 2011, S. 1508-1516.

15 Bertschi, Im Dazwischen von Individuum und Gesellschaft. Topologie eines blinden Flecks der Soziologie, Bielefeld 2010. 
mes aufscheinen. Weisser ${ }^{16}$ pointiert diese Beobachtungsgegenstände als sozialräumlich materialisierte Interessenkonflikte, d.h. als Situationen, in denen das Vermögen, etwas zu realisieren, ungleich verteilt ist, weil nicht alle Fähigkeiten gleich gefragt sind, nicht alle Chancen gleich eröffnet bzw. nicht alle Ressourcen gleich vorhanden, zugänglich und nutzbar sind. Diese Konflikte zwischen dem, was (für jemanden) gerade möglich, und dem, was gerade gefordert ist, werden beobachtbar im Feld der Verhältnisse und des Verhaltens.

Schon in den 1980er Jahren wurde - bewusst in Spannung zu damaligen integrationspädagogischen Forderungen nach Dezentralisierung und Regionalisierung der Hilfen - eine Debatte geführt um „Orte zum Leben“ für gesellschaftlich Ausgeschlossene. Der deutsche Protagonist Gaed ${ }^{17}$ schrieb der Behindertenhilfe ins Pflichtenheft, solche Orte zu schaffen für die häufig als strukturelle Verlierer einer mehr und mehr binnendifferenzierten und individualisierten Gesellschaft erscheinenden behinderten Menschen. Nicht in den Gemeinden sollten sie freigesetzt und damit ausgesetzt leben (müssen), sondern in speziell für sie zugeschnittenen Heimaten der traditionellen Leistungsanbieter, wie Diakonie oder Caritas. Aus der Perspektive der Anbieter dieser professionell geführten Schon- oder Förderräume sollte dort ein unverantwortlicher Entzug von Ressourcen für Schutzbedürftige verhindert werden, wogegen inklusives Planen und Denken Personen, die gerade durch ihre soziale Abhängigkeit charakterisiert seien, dem freien Spiel der Kräfte aussetze und so ihre Eigenentwicklung und ihre gesellschaftliche Wertschätzung gefährde (d.h. sie unangemessen gleich behandele).

Repräsentative Studien zeigten allerdings bereits zeitgleich, dass sich die zu Orten der Einbindung und Einbettung stilisierten Komplexeinrichtungen, in denen sich Einschränkungen und Abweichungen von gesellschaftlichen Leistungs- und Verhaltenserwartungen auf behinderte Menschen nicht negativ auswirken sollten, gerade als Organisationen mit hohem Regulierungs- und Reglementierungspotenzial gegenüber den dort Platzierten und Tätigen entpuppten, also ihre selbstbestimmte Teilhabe limitieren ${ }^{18}$.

$\mathrm{Ob}$ - jenseits solcher seit Jahrzehnten etablierten Konfliktlinien - mit Hilfe des Fachkonzepts der Sozialraumentwicklung nach objektiven Kriterien für verwirklichte Teilhabechancen gesucht werden kann, verdient daher wissenschaftlicher Aufmerksamkeit. Denn das Sozialraumkonzept will Divergenz und Differenz ebenso einbeziehen wie auf neue Steuerungsmechanismen für ein Leben in der Gemeinschaft aller achten ${ }^{19}$. Damit wäre es mehr als ein reines „Zuteilungsverfahren“ mit dem eingeschränkten

16 Weisser, Sozialraumorientierung und Situationen der Behinderung - Über die sozialräumliche Strukturierung von Abhängigkeitsbeziehungen, VHN 79/2010, S. 4, 6.

17 Gaedt, Einrichtungen für Ausgeschlossene oder „Ein Ort zum Leben“. Überlegungen zur Betreuung Geistigbehinderter, Jahrbuch für kritische Medizin Bd. 7, Berlin 1981, S. 96-109.

18 Wacker/Wetzler/Metzler/Hornung, Leben im Heim. Angebotsstrukturen und Chancen selbständiger Lebensführung in Wohneinrichtungen der Behindertenhilfe, Schriftenreihe des BMG 102, BadenBaden 1998.

19 So Hinte (Fußn. 14), S. 25. 
Blick auf Fall, stationäre oder ambulante Immobilie oder leistungssystematische $\mathrm{Zu}$ ständigkeit im territorialen Bezug. ${ }^{20}$

Kompliziert wird die erforderliche Kriteriensuche allerdings, weil gewissermaßen neue Erkenntniswege zu graben sind, wenn nicht nur eine bestehende kommunale Wirklichkeit abgebildet und als Bezugspunkt genutzt werden soll, sondern es darum geht, die zukünftigen Chancen und Grenzen der gesellschaftlichen Teilhabe bei Behinderung zu bemessen. Derzeit verstellen hierfür traditionelle Versorgungsmechanismen eher den Blick, und gute Beispiele der Teilhabe, die Schule machen und Transformationen bewirken könnten, sind rar.

Wie bei Emanzipationsprozessen nicht unüblich, muss also das Zusammenspiel von individueller Agency (Handlungspotenzial ${ }^{21}$ ) und sozialen Strukturen (d.h. auch der Sozialpolitik und des Sozialrechts) erfolgen unter Bedingungen der Ungleichzeitigkeit, insofern Akteure und Akteurinnen einbezogen sind, die derzeit in ihrem Zugang zu Ressourcen behindert sind und deren Verschiedenheit benachteiligt wird. Denn derzeit sind Menschen mit Beeinträchtigung eher gehalten, ihre Verschiedenheit zu verleugnen oder zu reduzieren (d.h. so „normal“ wie möglich zu erscheinen), um uneingeschränkt im sozialen und kommunalen Feld (und dort in Systemen wie Bildung, Mobilität, Politik etc.) teilhaben zu können.

Transformation ist also erforderlich, d.h. ein wesentlicher Wandel zu einer inklusiven Gesellschaft. Sie soll sich vollziehen durch die

- Institutionalisierung und Konsolidierung neuer, auf Inklusion gerichteter Leistungssysteme,

- Veränderung eines bestehenden defizitorientierten Bildes über beeinträchtigte Menschen und Selbstbildes beeinträchtigter Menschen,

- Entwicklung neuer Handlungs- und Deutungsmuster,

- Umformung von Strukturen und Architekturen und

- Umsetzung völkerrechtlicher Verträge in nationales Recht (insbesondere der UNBRK).

Ein erster Schritt wird sein, jenseits des Warenkorbs des bestehenden Nationalen Aktionsplans und vieler Landesaktionspläne, nicht nur eine klare Zielorientierung auf Inklusion anzubahnen, sondern dies auch mit einer Neudeutung bestehender Denkstrukturen über Behinderung zu verbinden. Der schrittweise Umbau vieler konzeptioneller, struktureller und lokaler Elemente muss gelingen, um den Übergang aus exkludierenden Kulturen und den neuen Zugang zu förderlichen Ressourcen im Alltag für beeinträchtigte Menschen zu eröffnen, d.h. zu Macht, Geld und Sinn.

20 Siehe die Debatte um ein „Sozialraumbudget“ zur Bearbeitung sämtlicher in einem Gebiet anfallenden Leistungsansprüche (nach $\S \S 27$ ff. SGB VIII), damit ein effektiverer und effizienterer Mitteleinsatz gewährleistet wird und auch präventive und auf Umgebungen (Netze) bezogene Entwicklungen finanziert werden können (Hinte (Fußn. 14), S. 26).

21 Bandura, Social cognitive theory: An agentic perspective, Annual Review of Psychology 52/2001, S. 1-26. 
Abb. 1 Ein Beispiel für Transformation: ESCHER „Luft und Wasser“ 22

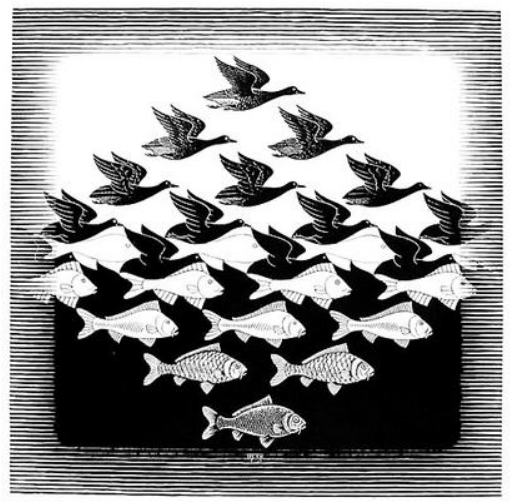

Inwiefern sich diese Transformationsprozesse durch Sozialraumorientierung vollziehen können, ist bislang kaum erforscht oder erprobt. Die Stärke einer Orientierung am sozialen Raum könnte im Blick auf Verbindungen und Interdependenzen liegen, die zugleich im transdisziplinären Diskurs (z.B. u.a. von Betriebswirtschaft, Gesundheitswissenschaft, Raumplanung, Sozialrecht und -politik) reflektiert werden können. Hier würde, bezogen auf den Personenkreis behinderter Menschen, weitgehend Neuland beschritten.

Hilfreich könnte die weltweit institutionalisierte Grammatik für den Verständigungsprozess darüber sein, Beeinträchtigungen nicht als „Eigenschaft einer Person“, sondern als Teil der menschlichen Verschiedenheit und Vielfalt zu verstehen. Dies ist verfasst in der Internationalen Klassifikation von Funktionsfähigkeit, Behinderung und Gesundheit (der ICF), wie sie von der WHO ${ }^{23}$ seit dem Jahr 2001 vorliegt. Dieses als ,bio-psychosozial“" bezeichnete Modell betrachtet komplexe (Wechsel-)Wirkungszusammenhänge der körperlichen, individuellen und gesellschaftlichen Dimensionen einer Behinderung und kann auch als international einsetzbare Richtschnur für die notwendigen Monitoringverfahren im Gesundheitssystem und der Rehabilitation dienlich sein.

Mit den Domänen der ,International Classification of Functioning, Disability and Health" 24 wird ein neuer multiperspektivischer Behinderungsbegriff operationalisierbar, der die sozialrechtlich üblichen Definitionen überschreitet. Es liegt daher nahe, sie als Transformator auf dem Weg zur geplanten und gestalteten neuen Community zu nutzen, weil sie nicht nur Behinderung sichtbar werden lässt, sondern auch einem über räumliche Perspektiven hinausreichenden Sozialraumbegriff genügt. Wesentlich ist der multiperspektivische Zuschnitt, der auch komplexe Lagen erfassen und aufdecken kann:

22 http://www.kunstkopie.de/a/escher-mc/luftundwasser8.html.

23 WHO - World Health Organization, International Classification of Functioning, Disability, and Health (ICF), Geneva 2001. Dt.: DIMDI - Deutsches Institut für Medizinische Dokumentation und Information (Hrsg.), Internationale Klassifikation der Funktionsfähigkeit, Behinderung und Gesundheit, Genf 2005, http://www.dimdi.de/static/de/klassi/icf/index.htm (Zugegriffen: 02. Januar 2013).

24 WHO (Fußn. 23). 
- Neben einer körperbezogenen Perspektive (body perspective) bietet sie

- eine personenbezogene Perspektive (individual perspective) sowie

- eine gesellschaftsbezogene Perspektive (societal perspective).

Insofern werden jeweils Körperfunktionen und -strukturen bzw. deren Beeinträchtigung (body functions and structures), Handlungsräume bzw. deren Einschränkungen (domains of activity, d.h. etwas tun können - capacity - und tun - performance) und Teilhabechancen bzw. deren Begrenzungen (domains of participation) betrachtet, was bereits die Aufmerksamkeit für deren komplexe und dynamische Interaktionen und Interrelationen schärft.

Abb. 2 International Classification of Functioning, Disability and Health ${ }^{25}$

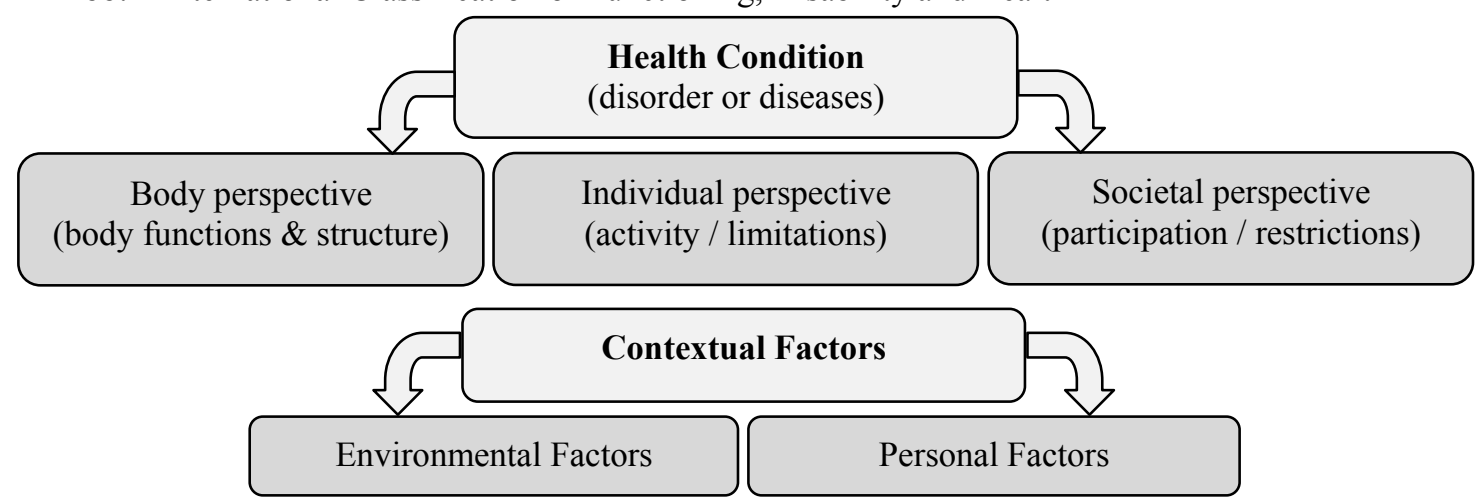

Schließlich geht es um die sog. Kontextfaktoren, die die möglichen Wechselbeziehungen zwischen Funktionalität, Aktivität und Partizipation in der Perspektive der jeweiligen Person und ihrer Umwelt abbilden sollen und damit aufzeigen, dass sie kontextsensibel, relativ und relational sind. Der Lebenshintergrund einer Person, der förderlich oder hinderlich wirken kann, gewinnt somit erhebliche Bedeutung, über eine reine Betrachtung von (chronischen) Krankheiten oder Funktionsstörungen hinaus.

Bei den Umweltfaktoren (environmental factors) ist die materielle, soziale und einstellungsbezogene Umwelt gemeint, in der Menschen leben und die ihr Leben mitgestaltet. Umweltbezogene Elemente sind konkret

- die dingliche Umwelt: Produkte und Technologien (z.B. Hilfsmittel, Barrieren im Lebensumfeld, Medikamente),

- die natürliche und vom Menschen veränderte Umwelt (in Form von Räumen, Gebäuden, Verkehrswegen),

- die soziale Umwelt: Angehörige, Partnerinnen bzw. Partner und Familienkreis (bis zu Haustieren), Freunde und Bekannte, Unterstützungs- und Pflegepersonen, medizinische, therapeutische, pädagogische, psychologische und andere Fachleute und die Beziehung zu ihnen wie Assistenz, Begegnung, Beratung, Diagnose, Fürsorge, „Gate keeping“, Therapie etc.

25 WHO (Fußn. 23). 
- die kulturelle Umwelt: also die weitgehend verschlungenen Einstellungen, Urteile, Bewertungen/Beurteilungen, Rollen, gesellschaftlichen Normen, Konventionen und Weltanschauungen sowie schließlich

- die Leistungssysteme: soziale Sicherungssysteme und -dienste und deren Konzepte, das Gesundheitswesen, Gesundheits- und Sozialpolitik, Selbsthilfesysteme, das Bildungs-, Arbeits- und Beschäftigungswesen und seine Träger.

Die personenbezogenen Faktoren (personal factors) hingegen nehmen Bezug auf Gegebenheiten der Person, ihr spezifisches Leben, ihre Erfahrungen sowie individuellen Strategien der Lebensführung und Aufgabenbewältigung. Im Einzelnen werden Aspekte beachtet wie

- Alter,

- Geschlecht,

- ethnische Zugehörigkeit bzw. Zuwanderungserfahrung, aber auch Ressourcen wie

- Erziehung,

- Bildung bzw. Ausbildung,

- Beruf und

- sozialer Hintergrund bzw. Herkunft.

Weitere Charakteristika und Verhaltensmuster, wie Lebensstile und Gewohnheiten, können Risikofaktoren oder Ressourcen sein; vergangene und gegenwärtige Erfahrungen, soziale Kompetenzen und Fähigkeiten (wie Adaptation, „Coping“, Empathie, Flexibilität, Motivation, Vitalität), physische Fitness und psychisches Leistungsvermögen 26 .

Die ICF ist also denkbar als der maßgebliche internationale und fachübergreifende Standard, um Gesundheit und Beeinträchtigung bzw. die Behinderung einer Person (individuelle Ebene) oder in einer Bevölkerung (gesellschaftliche Ebene) zu erfassen und zu beschreiben. Sie beleuchtet im Alltagsbezug die fließenden Grenzen zwischen Gesundheit und Beeinträchtigung (salutogenetischer Ansatz ${ }^{27}$ ) und verdeutlicht die Vielfalt und Verschiedenheit der Beeinträchtigungserfahrungen als Element des Menschseins. Ausgangspunkt ist nicht mehr der Fähigkeitsmangel einer einzelnen Person, sondern sind die Wirkungen verschiedener Umstände der Fähigkeitsentfaltung in konkreten Lebensräumen (from cause to impact). Dadurch treten vor allem die sozialen Aspekte von Behinderung in den Vordergrund, gegenüber einer bio-medizinischen Perspektive von Dysfunktionen. Auch der Blick auf das Leistungssystem wandelt sich. Maßnahmen werden neu bewertet vor der Ausrichtung an einem kommunalen Leistungsgefüge der

26 Schuntermann, Einführung in die ICF: Grundkurs, Übungen, offene Fragen, Hamburg 2005.

27 Antonovsky, Salutogenese: zur Entmystifizierung der Gesundheit (Dt. erw. Hrsg. Franke), Tübingen 1997. 
Rehabilitation (community-based long-term services) ${ }^{28}$. Im Einzelnen geht es um jeweils passende konkrete Wohn- und Lebensverhältnisse, um den Abbau von Barrieren und die Bereitstellung von Hilfsmitteln, um Maßnahmen und Informationen zur Prävention, um geeignete therapeutische Interventionen und Rehabilitation im Lebensumfeld, aber auch um Möglichkeiten der selbstbestimmten Mobilität in der Häuslichkeit und im öffentlichen Raum, um Unterstützungsbedarfe und -bedürfnisse, um verfügbare Assistenz durch Angehörige oder andere relevante Personen (Nachbarn, Freunde, Vereine), um mögliche Selbsthilfepotenziale sowie Bürgerschaftliches Engagement; es geht ebenso um das sozialrechtliche Umfeld (bestehende Leistungsansprüche und den tatsächlichen Zugang zu Leistungen), um wirtschaftliche Rahmenbedingungen, um den Zugang $\mathrm{zu}$ und die Nutzung von Information und Kommunikation (Beratung, Medien). Zugleich werden im Bereich Bildung, Ausbildung und Beruf sowohl die Regel- und Sonder/Fördersysteme (Frühförderung, Schulen, Berufsbildungswerke, Berufsförderungswerke, Werkstätten für behinderte Menschen, der Zugang zum Dualen Bildungssystem und zu Hochschulen) als auch die Chancen auf dem allgemeinen Arbeitsmarkt und der Bestand sowie die Wirkung von angepassten Arbeitsplätzen und Integrationsdiensten in den Blick genommen. Dies alles soll ebenso berücksichtigt werden, wie die Vielfalt der Beeinträchtigungsarten und Verschiedenheit ihrer Ausprägung und Bedeutung für konkrete Personen.

\section{III. Überall - „Mainstreaming Disability“ als Entwicklungsprogramm und-methode}

Der differenzierte Auftrag, im Lebensraum aller (also auch dem kommunalen Raum bzw. dem Quartier) die Lebenschancen der Menschen mit Beeinträchtigung so beschreiben und bewerten zu können, dass Ansatzpunkte für den Prozess einer Entwicklung zur inklusiven Gesellschaft evident werden, wurde reflektiert und operationalisiert. In einem ,funktional ausdifferenzierten, demokratischen Sozialstaat[s] wird deutlich, dass Inklusion kein ausschließliches Thema für oder über behinderte Menschen ist, sondern ein rechtlich gesichertes Prinzip der sozialen Einbeziehung der Bevölkerung" 29 in Form der Abkehr von rein maßnahmen- und institutionenbezogenem Denken und Handeln ${ }^{30}$. Das Gemeinwesen als „welcoming community“ soll Zugehörigkeit aller garan-

28 Lightfood, Community-based rehabilitation. A rapidly growing method for supporting people with disabilities, Social Work 47/2004, S. 455-468; Scholz, Die Entwicklung des Community CareAnsatzes in Deutschland und Großbritannien im Vergleich, Norderstedt 2009.

29 Wansing, Der Inklusionsbegriff in der Behindertenrechtskonvention, in: Welke (Hrsg.), UNBehindertenrechtskonvention mit rechtlichen Erläuterungen, Berlin 2012, S. 93, 95.

30 Rat der Europäischen Union, Ziele bei der Bekämpfung der Armut und der sozialen Ausgrenzung (Dokument 14164/1/02 REV 1), Brüssel 2002. 
tieren, auch der behinderten Menschen, aber zukünftig in neuer Form, also nicht mehr als ihre institutionelle Ausgrenzung in der Gesellschaft ${ }^{31}$.

Die Konkretion solcher Überlegungen macht nicht nur Wirkungszusammenhänge sichtbar, sondern auch eine ausgeprägte Komplexität durch die Vielfalt relevanter Items und Denkmodelle. Alleine die menschliche Verschiedenheit ebenso wie die Heterogenität dessen, was als Behinderung gilt, ist erheblich. Es ist daher eine Fiktion, von „den Behinderten“ auszugehen, ohne die Diversität in Alter, Geschlecht, Lebensumständen, Eintritt der Beeinträchtigung, Zugang zu Ressourcen und vieles mehr zu berücksichtigen. Scheinbar im „Überall“ angekommen, liegt nun allerdings die Frage nahe, ob nicht durch die Suche nach Chancengerechtigkeit bei Beeinträchtigung im Sozialraum vor allem eine wissenschaftliche Enttäuschung entdeckt wird: nämlich eine Überkomplexität, die Evidenz kaum möglich macht. Lohnt es dann überhaupt, die eingangs angestrebte Suche fortzusetzen, um mögliche Schätze für ein wissenschaftsbasiertes Entwicklungskonzept zu heben?

Hier soll zu Hartnäckigkeit ermuntert werden. Denn vermutlich gibt es relevante Interdependenzen! Solche Cluster zu identifizieren, wäre ein erster Schritt. Die zu erwartende Komplexität wird wohl die des vor über drei Jahrzehnten ersonnenen magischen Würfels des ungarischen Architekten und Bauingenieurs Rubik nicht unterschreiten, der angeblich einen Anreiz geben wollte, räumliches Denkvermögen zu trainieren. Vielleicht könnte die ICF in Kombination mit Sozialraumentwicklungen zumindest ähnliche Anreize geben, vielleicht geht aber auch mehr!

Denn die Zielorientierung für erforderlichen Wandel ergibt sich aus der Vision der Inklusion, die die UN-BRK als weitere Leitstruktur ins Spiel bringt: als dritte Dimension für die anstehenden Transformationsaufgaben. Die aus der UN-BRK abgeleitete Aufgabe, Behindertenpolitik in allen politischen Gestaltungsfeldern als Querschnittelement zu konkretisieren (sog. Disability Mainstreaming) ${ }^{32}$, eröffnet dann Chancen, dass faktisch bestehende Bedarfe und Bedürfnisse der Menschen, die mit Beeinträchtigungen leben, nicht „,im Übergang“ verlorengehen ${ }^{33}$.

31 Kronauer, „Exklusion“ als Kategorie einer kritischen Gesellschaftsanalyse. Vorschläge für eine anstehende Debatte, in: Bude/Willisch (Hrsg.), Das Problem der Exklusion. Ausgegrenzte, Entbehrliche, Überflüssige, Hamburg 2006, S. 27, 41; ebenso Nassehi, Die paradoxe Einheit von Inklusion und Exklusion. Ein systemtheoretischer Blick auf die „Phänomene“, in: Bude/Willisch (Hrsg.) (Fußn. 31), S. 46, 49 ff.

32 Vgl. Aselmeier, Community Care und Menschen mit geistiger Behinderung, Wiesbaden 2008.

33 Wacker, Behindertenpolitik, Behindertenarbeit, in: Otto/Thiersch (Hrsg.), Handbuch Soziale Arbeit, 4. völlig neu bearbeitete Aufl. München \& Basel 2011, S. 87-100. 
Abb. 3 Lebenschancenansatz

\section{Lebenschancenansatz}

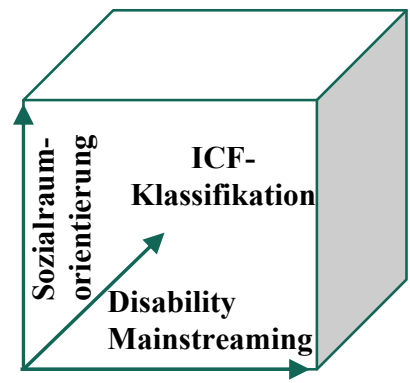

Die UN-BRK artikuliert bezogen auf Behinderung neue Daueraufträge an die Unterzeichner. In der Präambel findet sich die Mission, Behinderungsumstände ständig und dauerhaft als Bestandteil gesellschaftlicher Entwicklungsprozesse zu berücksichtigen: „Emphasizing the importance of mainstreaming disability issues [Hervor. Verf.] as an integral part of relevant strategies of sustainable development" [Buchstabe g] [...].

Zugleich werden Wege aktiver partizipativer Entwicklung und Umsetzung entsprechender politischer Konzepte und Programme festgelegt: „Considering that persons with disabilities should have the opportunity to be actively involved in decisionmaking processes about policies and programmes, including those directly concerning them" [Buchstabe o], [...].

Auch die hiermit erforderliche neue systematische Betrachtung der Zusammenhänge zwischen UN-BRK und Disability Mainstreaming steht noch aus. Hier - wie auch bezogen auf Leistungsansprüche - sind viele Fragen offen und Metamorphosen im Leistungsgeschehen erforderlich. Klar ist aber bereits: Jede genutzte Stellschraube wird zu weiteren Veränderungen der Wirklichkeit von Behinderung in der Gesellschaft führen. Also wird Forschung zu Teilhabe auch einem Geduldspiel gleichkommen, bei dem jede Variation neue Ausgangslagen herbeiführt und man in kleinen Segmenten seinen Forschungsgegenstand jeweils neu beleuchten muss, um neue Passungen zu entdecken.

Grundsatzfragen sind u.a.

- der Umgang mit Vielfalt und Verschiedenheit bei Beeinträchtigung (Diversity Approach und Disability Mainstreaming),

- Möglichkeiten der Selbstbestimmung und Teilhabe,

- die Bedeutung und der Einfluss des demografischen Wandels,

- die Konstruktionen sozialer Sicherung und ihre Möglichkeiten und Grenzen,

- erforderliche neue Dienste und Rollen im gewandelten Unterstützungssystem,

- Relevanz von Subsidiarität und neue (nutzerorientierte) Steuerung der Leistungen,

- Diffusion gewandelter Leitbilder und rechtlicher Rahmensetzungen auf nationaler und internationaler Ebene, 
- Ausprägung von Lebensqualität und Wohlbefinden bei Beeinträchtigung,

- Aufbau generell unterstützender Umwelten (Universal Design bzw. Design für alle) sowie die Entwicklung spezifisch assistiver Technologien (Ambient Assisted Living bzw. selbstbestimmtes Leben durch assistierende Technologien).

Einer wirkungsorientierten Prüfung müssen aber auch Fragen standhalten, wie traditionell institutionell versorgte beeinträchtigte Personen ihren Willen entwickeln, erkennen und einbringen können in einer Weise, die nicht überlagert ist von ihren bisherigen und aktuellen Möglichkeiten und Grenzen der Lebenserfahrung, von gewohnten restriktiven Handlungsspielräumen in „All inclusive-Settings“ und vom Mangel an Alternativen im Unterstützungsgeschehen. Aus anderer Perspektive beleuchtet, geht es um Fragen, wie umfänglich professionelle Kompetenzen, Spezialqualifizierungen und -angebote in sozialräumlichen Ausformungen erforderlich sind und Einfluss nehmen auf eigene Konzepte und Lösungen behinderter Menschen. Im Licht der Subsidiarität ist zu fragen, welche gesellschaftlichen Erwartungen an beeinträchtigte Menschen als engagierte Bürgerinnen und Bürger zu richten wären und wie Grenzen ihrer Eigenleistung oder der Leistungen ihrer sozialen Netze auszuloten sind. Und sieht man aus der Perspektive möglicher Effektivität und Effizienz des Ressourceneinsatzes auf die verschiedenen parallel funktionierenden und agierenden Sozialleistungssysteme, drängt sich die Frage auf, wie nicht oder kaum genutzte systemüberschreitende Kooperationschancen und Pfadleitungen (durch passgenaue Beratung und nutzbare Information und Kommunikation) trägerübergreifend wachsen können in kommunalen Territorien.

Aus dem Blickwinkel gewünschter Lebensqualität und erfahrenen Wohlbefindens sind neben grundsätzlichen Ausstattungen für ein gesundes Leben, die am Zugang zu Grunderfordernissen, wie Sicherheit, Nahrung, Schutz, gesunde Umwelt etc., für alle festzumachen sind, auch neue Konzepte erwägenswert:

- Neue soziale Gemeinschaften, wie sie sich beispielsweise im Internet entwickeln, vernetzen soziale Interessen und haben längst den Status des Nischendaseins verlassen; „smart“ organisierte Fahrräder, Autos oder Transaktionen werden Alltag, ebenso könnten sich Gesundheits-, Mobilitäts- und soziale Dienste entwickeln.

- Personenzentrierte Ansätze, wie individuelle Teilhabeplanung, individuelle Leistungsansprüche und -gestaltung im Leistungsgeschehen, entstehen vor $\mathrm{Ort}^{34}$, ebenso könnten kommunale Gesundheitslandschaften entstehen, in denen bei Beeinträchtigung passende Versorgungsstrukturen partizipativ gestaltet und verfügbar werden.

34 Lübbe/Beck, Individuelle Hilfeplanung - Anforderungen an die Behindertenhilfe, Bremen 2002; Rund/Lutz/Fiegler, Kommunale Teilhabeplanung im Kontext Integrierter Sozialraumplanung, in: Lampke/Rohrmann/Schädler (Hrsg.), Örtliche Teilhabeplanung mit und für Menschen mit Behinderungen. Theorie und Praxis, Wiesbaden 2011, S. 89-104. 
Neue kommunale Ermöglichungsräume (als „enabling communities“, befähigte und befähigende Gemeinschaften ${ }^{35}$ ) zu realisieren, wird eine Aufgabe der kommenden Jahre $\operatorname{sein}^{36}$. Dies erscheint in weiten Strecken kongruent mit dem Ziel, die Infrastruktur wohnortnah und gemeindebasiert mit den Erfordernissen einer wachsenden älteren Bevölkerung in Einklang zu bringen ${ }^{37}$. Mit Nassehi ${ }^{38}$ könnte man dafür neu fragen, nicht, „wer die Exkludierten sind, wer die Überflüssigen und wer die Ausgegrenzten. Vielleicht ist es angemessener danach zu fragen, unter welchen Bedingungen sich Lebenslagen als exkludiert, überflüssig, ausgegrenzt beschreiben.“.

In dem künftigen Gemeinwesen sollten auch beeinträchtigte Bürgerinnen und Bürger selbstbestimmt leben und dabei in die Gemeinschaft einbezogen sein (Article 19 UNBRK „Living independently and being included in the community“). Allen müssen die gleichen Wahlmöglichkeiten offenstehen, die dafür notwendigen wirksamen und geeigneten Vorkehrungen sind $\mathrm{zu}$ treffen (,take effective and appropriate measures to facilitate full enjoyment by persons with disabilities of this right and their full inclusion and participation in the community"). Dabei ist an Elemente gedacht wie

- die Wahl des Aufenthaltsortes, der Wohnform und der Personen, mit denen man lebt (a),

- Zugang zu Diensten in der Gemeinde (auch als persönliche Assistenz) (ohne Risiken der Isolation und Aussonderung) (b),

- Dienste für alle in der Gemeinde, die den Bedürfnissen der Gleichberechtigung bei Behinderung Rechnung tragen (c).

Wie solche Transformationen trotz teilweise konträrer Dynamiken einzelner jahrzehntelang etablierter Leistungserbringer und -anbieter aus Altenhilfe, Eingliederungshilfe, Kinder- und Jugendhilfe, Pflegesicherung etc. und regionaler sowie überregionaler Wirkkräfte gelingen können, wird aufwändig zu konzipieren und wissenschaftlich zu prüfen sein, wie dies auch von Beginn des Transformationsprozesses von Sach- zu Geldleistungssystemen an geschah (Monitoring der Entwicklung und Umsetzung des Persönlichen Budgets nach § 17 SGB IX $\left.{ }^{39}\right)$.

35 Treber/Haas, Enabling Community. Gemeinwesen zur Inklusion befähigen, in: Schütte (Hrsg.), Abschied vom Fürsorgerecht. Von der „Eingliederungshilfe für behinderte Menschen“ zum Recht auf soziale Teilhabe, Münster, Hamburg \& London 2011, S. 141, 142.

36 Kreutz/Lachwitz/Trenk-Hinterberger, Die UN-Behindertenrechtskonvention in der Praxis. Erläuterungen der Regelungen und Anwendungsgebiete, Köln 2013; Steinhart, Teilhabe für alle im Quartier. Herausforderungen für die Sozialpsychiatrie, in: Aktion Psychisch Kranke/Weiß (Hrsg.), Psychiatriereform 2011 ... Der Mensch im Sozialraum, Bonn 2012, S. 52-68; Welti, Rechtliche Grundlagen einer örtlichen Teilhabeplanung, in: Lampke/Rohrmann/Schädler (Hrsg.) (Fußn. 34), S. 55-67.

37 Barth, Örtliche Teilhabeplanung für ältere Menschen mit und ohne Behinderung, in: Lampke/Rohrmann/Schädler (Hrsg.) (Fußn. 34), S. 257-268.

38 Nassehi (Fußn. 31), S. 68.

39 Schäfers/Wacker/Wansing, Persönliches Budget im Wohnheim, Wiesbaden 2009; Wacker, Das Persönliche Budget. Neue Leistungsgestaltung in der Behindertenhilfe, Die Rehabilitation 48/2009, S. 4-14; Wansing, Persönliches Budget, in: Greving (Hrsg.), Kompendium Heilpädagogik. Bd. 2, Troisdorf 2007, S. 165-175. 


\section{Ausblick-evidenzbasierte Maßstäbe für Leistungssysteme als}

\section{Königsweg?}

Die Verwebungen und Verflechtungen bei Beeinträchtigung und Behinderung auf der Landkarte der Lebenslagen und Lebenswelten sind vermutlich relevant und kaum weniger komplex und herausfordernd als die Netze und mehrdimensionalen Verbindungen, die systembiologisch mit Hilfe von Hochleistungscomputern aufgedeckt werden sollen. Zwischen molekularem Minimaß und Gesamtorganismen verbergen sich charakteristische Muster, die Leben bedeuten. Mit ebendiesen systemtheoretischen Logiken will Sozialwissenschaft der Gesellschaft auf die Spur ihrer Konstruktionen und regulatorischen Prozesse kommen.

Schon Kant betrachtete Lebewesen als ,organisierte und sich selbst organisierende Wesen“40. Hieraus ergeben sich Perspektiven und mögliche Folgen, denen sich sozialpolitisch und sozialrechtlich fokussierte Forschung nicht entziehen kann, noch kann sie sich auf einen unspezifischen Begriff der Vernetzung zurückziehen. Wie gezeigt wurde, müssen geeignete Heuristiken noch identifiziert werden, die bei den Rekonstruktionen sozialer Verflechtungen ein Abbild des Alltags ermöglichen ${ }^{41}$. Dies könnte Aufgabe der Teilhabeforschung sein, die Modelle entwickelt und konkrete Situationen erkennt und benennt, die Zusammenhänge zwischen Innen und Außen der Inklusion und Exklusion, ihrem Wesen und ihrer Erscheinung, aufdeckt, beschreibt und prüft. Als ein Indikator kann die ICF-Systematik wertvolle Anhaltspunkte bereitstellen, obwohl Alltag vielfach komplexe Feinstrukturen enthält.

Widerstände sind zu erwarten, weil sich Gesellschaft durch Exklusion auch funktionsfähig hält und lokale Strukturen kein Allheilmittel sind. Einerseits wird vor kommunalen „Ghettos ohne Mauern“ für multipel und kumuliert Benachteiligte gewarnt ${ }^{42}$, andererseits die Nicht-Unterscheidbarkeit tatsächlicher Leistungsansprüche gegenüber gesellschaftspolitischen Desideraten sozialer Kontextbezüge als ,,aus der Flasche gelassene[r] Geist" heraufbeschworen 43 .

Diese Einwände mögen als Hinweis dienen für manche Mühsal, mit der Prozesse zur Implementierung bzw. Transformation gerechter Lebenschancen für behinderte Menschen im Gemeinwesen verbunden sind ${ }^{44}$. Konkrete Maßstäbe im Prozess des evidenz-

40 Zit. nach Rheinberger, Biologie - mit System neu definiert, Max Planck Forschung H. 1/2012, S. 12-16.

41 Cordts, Örtliche Teilhabeplanung als Herausforderung für vertikale und horizontale Kooperationsprozesse beteiligter Leistungsträger und Anbieterorganisationen, in: Lampke/Rohrmann/Schädler (Hrsg.) (Fußn. 34), S. 111-126.

42 Häußermann, Die Krise der ,sozialen Stadt“. Warum der sozialräumliche Wandel der Städte eine eigenständige Ursache für Ausgrenzung ist, in: Bude/Willisch (Hrsg.) (Fußn. 31), S. 294, 311.

43 Luthe, Begriff und Gegenstand des Rehabilitationsrechts, SGb 8/07, S. 454, 461.

44 Etzioni, Die Entdeckung des Gemeinwesens - Das Programm des Kommunitarismus, Frankfurt a.M. 1998. 
basierten Monitoring und Vergleichens wären hilfreich, jenseits allen guten Willens. Stein ${ }^{45}$ bezeichnet Inklusion ,als Utopie, aber nicht im Sinne eines Unerreichbaren, sondern als U-topos: als Noch-nicht-Ort“. Wegweisungen können aus der systematischen Analyse von Ausgrenzungsprozessen und bei der Fahndung nach Kraftquellen und Möglichkeitsräumen aufscheinen. Dies ist eine gesellschaftliche Frage und Aufgabe, bei der sowohl individuelle Unterstützungsbedarfe eingelöst werden, als auch nicht aussondernde gesellschaftliche Strukturen entstehen.

Für eine im immer neuen Übergang erforderliche praktische Utopie der Teilhabe von behinderten Menschen, wird ein Handeln im Optativ den Weg bahnen, das von Schütz angedachte phantasievolle Durchspielen von Möglichkeiten ${ }^{46}$, das zu einer Anpassung der bestehenden und sich entwickelnden Entwürfe im Handlungsvollzug führt. Somit wäre die Differenz zwischen dem, was wir dank unserer Vorstellungskraft vom Umgang mit Behinderung erwarten und dem, was geschieht, Quelle von Neuem. Das Neue entsteht in der Transformation des Alten. Wie viel Licht hier am Ende des Tunnels sein wird, muss sich finden!

\section{Literatur}

Antonovsky, Salutogenese: zur Entmystifizierung der Gesundheit (Dt. erw. Hrsg. Franke). DGVT, Tübingen 1997.

Aselmeier, Community Care und Menschen mit geistiger Behinderung, Wiesbaden 2008.

Baer/Bittner/Göttschke, Mehrdimensionale Diskriminierung - Begriffe, Theorien und juristische Analyse. Teilexpertise im Auftrag der Antidiskrimimierungsstelle des Bundes, Berlin 2011.

Bandura, Social cognitive theory: An agentic perspective, Annual Review of Psychology 52/2001, S. 1-26.

Barth, Örtliche Teilhabeplanung für ältere Menschen mit und ohne Behinderung, in: Lampke/Rohrmann/Schädler (Hrsg.), Örtliche Teilhabeplanung mit und für Menschen mit Behinderungen. Theorie und Praxis, Wiesbaden 2011, S. 257-268.

Bingel, Sozialraumorientierung revisited. Geschichte, Funktion und Theorie sozialraumbezogener Sozialer Arbeit, Wiesbaden 2011.

Bertschi, Im Dazwischen von Individuum und Gesellschaft. Topologie eines blinden Flecks der Soziologie, Bielefeld 2010.

Bude/Lantermann, Soziale Exklusion und Exklusionsempfinden, Kölner Zeitschrift für Soziologie und Sozialpsychologie 58/2006, S. 233-252.

45 Stein, Integration als Möglichkeitsraum der Vergesellschaftung von Individuen, Behindertenpädagogik 47/2008, S. 283, 288.

46 Knoblauch, Alfred Schütz, die Phantasie und das Neue. Überlegungen zu einer Theorie des kreativen Handelns, in: Schröer/Bidlo (Hrsg.), Die Entdeckung des Neuen. Qualitative Sozialforschung als Hermeneutische Wissenssoziologie, Wiesbaden 2011, S. 99, 105. 
Council of the European Union. Joint report by the Commission and the Council of social inclusion. Brussels 2004, p 10, SOC 115 ECOFIN 80 EDUC 46 SAN 49.

Cordts, Örtliche Teilhabeplanung als Herausforderung für vertikale und horizontale Kooperationsprozesse beteiligter Leistungsträger und Anbieterorganisationen, in: Lampke/Rohrmann/Schädler (Hrsg.), Örtliche Teilhabeplanung mit und für Menschen mit Behinderungen. Theorie und Praxis, Wiesbaden 2011, S. 111-126.

Dern/Inowlocki/Oberlies, Mehrdimensionale Diskriminierung - Eine empirische Untersuchung anhand von autobiographisch-narrativen Interviews. Teilexpertise im Auftrag der Antidiskriminierungsstelle des Bundes, Berlin 2011.

Etzioni, Die Entdeckung des Gemeinwesens - Das Programm des Kommunitarismus. Fischer, Frankfurt a.M. 1998.

Europäischer Kongress von Menschen mit Behinderung, Die Madrid-Deklaration. 23. März 2002, Madrid. Disability World. A bimonthly web-zine of international disability news and views Issue no. 15 September-October 2002. http://www.disabilityworld.org/0910_02/news/madrid.shtml (Zugegriffen: 02. Januar 2013).

Früchtel/Budde/Cyprian, Sozialer Raum und Soziale Arbeit. Textbook: Theoretische Grundlagen, Wiesbaden 2007.

Gaedt, Einrichtungen für Ausgeschlossene oder „Ein Ort zum Leben“. Überlegungen zur Betreuung Geistigbehinderter, Jahrbuch für kritische Medizin Bd. 7, Berlin 1981, S. 96-109.

Grüber, Disability Mainstreaming, IMEW konkret Nr. 10, Dezember 2007, abrufbar unter: http://www.imew.de/index.php?id=237 (letzter Zugriff: 19.03.2011).

Häußermann, (2006) Die Krise der „sozialen Stadt“. Warum der sozialräumliche Wandel der Städte eine eigenständige Ursache für Ausgrenzung ist, in: Bude/Willisch (Hrsg.), Das Problem der Exklusion. Ausgegrenzte, Entbehrliche, Überflüssige, Hamburg 2006, S. 294313.

Hinte, Eigensinn und Lebensraum - zum Stand der Diskussion um das Fachkonzept „Sozialraumorientierung“, VHN 78/2009, S. 20-33.

Kessl/Reutlinger, Sozialraum, in: Otto/Thiersch (Hrsg.), Handbuch Soziale Arbeit, 4. Aufl. München/Basel 2011, S. 1508-1516.

Knoblauch, Alfred Schütz, die Phantasie und das Neue. Überlegungen zu einer Theorie des kreativen Handelns, in: Schröer/Bidlo (Hrsg.), Die Entdeckung des Neuen. Qualitative Sozialforschung als Hermeneutische Wissenssoziologie, Wiesbaden 2011, S. 99-116.

Kreutz/Lachwitz/Trenk-Hinterberger, Die UN-Behindertenrechtskonvention in der Praxis. Erläuterungen der Regelungen und Anwendungsgebiete, Köln 2013.

Kronauer, „Exklusion“ als Kategorie einer kritischen Gesellschaftsanalyse. Vorschläge für eine anstehende Debatte, in: Bude/Willisch (Hrsg.), Das Problem der Exklusion. Ausgegrenzte, Entbehrliche, Überflüssige, Hamburg 2006, S. 27-45.

Lübbe/Beck, Individuelle Hilfeplanung - Anforderungen an die Behindertenhilfe, Bremen 2002.

Luthe, Begriff und Gegenstand des Rehabilitationsrechts, SGb 2007, S. 454-467.

Lightfood, Community-based rehabilitation. A rapidly growing method for supporting people with disabilities, Social Work 47/2004, S. 455-468.

Lutz/Wenning, Differenzen über Differenz - Einführung in die Debatten, in: Lutz/Wenning (Hrsg.), Unterschiedlich verschieden. Differenz in der Erziehungswissenschaft, Opladen 2001, S. 11-24. 
Minow, Making All the Difference. Inclusion, Exclusion and American Law, Ithaka/New York 1990.

Nassehi, Die paradoxe Einheit von Inklusion und Exklusion. Ein systemtheoretischer Blick auf die „Phänomene“, in: Bude/Willisch (Hrsg.), Das Problem der Exklusion. Ausgegrenzte, Entbehrliche, Überflüssige, Hamburg 2006, S. 46-69.

Rat der Europäischen Union, Ziele bei der Bekämpfung der Armut und der sozialen Ausgrenzung (Dokument 14164/1/02 REV 1), Brüssel 2002.

Rund/Lutz/Fiegler (2011). Kommunale Teilhabeplanung im Kontext Integrierter Sozialraumplanung, in: Lampke/Rohrmann/Schädler (Hrsg.), Örtliche Teilhabeplanung mit und für Menschen mit Behinderungen. Theorie und Praxis, Wiesbaden 2011, S. 89-104.

Rheinberger, Man weiß nicht genau, was man nicht weiß. Über die Kunst, das Unbekannte zu erforschen, NZZ, 5.5.2007.

Rheinberger, Biologie - mit System neu definiert, Max Planck Forschung H. 1 2012, S. 12-16.

Schäfers/Wacker/Wansing, Persönliches Budget im Wohnheim, Wiesbaden 2009.

Scholz, Die Entwicklung des Community Care-Ansatzes in Deutschland und Großbritannien im Vergleich, Norderstedt 2009.

Schütz, Der sinnhafte Aufbau der sozialen Welt. Eine Einleitung in die verstehende Soziologie, Frankfurt a.M. 1974.

Schütz/Luckmann, Strukturen der Lebenswelt, Bd. I, Frankfurt a.M. 1979.

Schuntermann, Einführung in die ICF: Grundkurs, Übungen, offene Fragen, Hamburg 2005.

Sen, Ökonomie für den Menschen, München 2007.

Sennett, Respekt im Zeitalter der Ungleichheit, Berlin 2004.

Steinhart, Teilhabe für alle im Quartier. Herausforderungen für die Sozialpsychiatrie, in: Aktion Psychisch Kranke/Weiß (Hrsg.), Psychiatriereform 2011 ... Der Mensch im Sozialraum, Bonn 2012, S. 52-68.

Stein, Integration als Möglichkeitsraum der Vergesellschaftung von Individuen, Behindertenpädagogik 47/2008, S. 283-298.

Treber/Haas, Enabling Community. Gemeinwesen zur Inklusion befähigen, in: Schütte (Hrsg.), Abschied vom Fürsorgerecht. Von der „Eingliederungshilfe für behinderte Menschen“ zum Recht auf soziale Teilhabe, Münster/Hamburg/London 2011, S. 141-161.

UN - United Nations, The Standard Rules on the Equalization of Opportunities for Persons with Disabilities. Adopted by the United Nations General Assembly, forty-eighth session, resolution 48/96, annex, of 20 December 1993. http://www.un.org/ga/search/view_doc.asp? symbol=A/RES/48/96\&Lang=E\&Area=RESOLUTION. Zugegriffen: 02. Januar 2013.

Voges/Jürgens/Mauer/Meyer, Methoden und Grundlagen des Lebenslagenansatzes, ZeS: Zentrum für Sozialpolitik Endbericht 2003 (http://www.soziologie.uni-kiel.de/bergersozun/ Voges_Lebenslagenansatz.pdf).

Wacker, Behinderung in der Gesellschaft. 50 Jahre im soziologischen Blick - vom Dialog zum Diskurs, Geistige Behinderung 47/2008, S. 42-61.

Wacker, Das Persönliche Budget. Neue Leistungsgestaltung in der Behindertenhilfe, Die Rehabilitation 48/2009, S. 4-14. 
Wacker/Wetzler/Metzler/Hornung, Leben im Heim. Angebotsstrukturen und Chancen selbständiger Lebensführung in Wohneinrichtungen der Behindertenhilfe, Schriftenreihe des BMG 102, Baden-Baden 1998.

Wacker, Behindertenpolitik, Behindertenarbeit, in: Otto/Thiersch (Hrsg.), Handbuch Soziale Arbeit, 4. Aufl. München/Basel 2011, S. 87-100.

Wansing, Persönliches Budget, in: Greving (Hrsg.), Kompendium Heilpädagogik, Bd. 2, Troisdorf 2007, S. 165-175.

Wansing, Der Inklusionsbegriff in der Behindertenrechtskonvention, in: Welke (Hrsg.), UNBehindertenrechtskonvention mit rechtlichen Erläuterungen, Berlin 2012, S. 93-103

Welti, Rechtliche Grundlagen einer örtlichen Teilhabeplanung, in: Lampke/Rohrmann/Schädler (Hrsg.), Örtliche Teilhabeplanung mit und für Menschen mit Behinderungen. Theorie und Praxis, Wiesbaden 2011, S. 55-67.

Weisser, Sozialraumorientierung und Situationen der Behinderung - Über die sozialräumliche Strukturierung von Abhängigkeitsbeziehungen, VHN 79/2010, S. 4-10.

WHO - World Health Organization, International Classification of Functioning, Disability, and Health (ICF), Geneva 2001. Dt.: DIMDI - Deutsches Institut für Medizinische Dokumentation und Information (Hrsg.), Internationale Klassifikation der Funktionsfähigkeit, Behinderung und Gesundheit, Genf 2005, http://www.dimdi.de/static/de/klassi/icf/index.htm (Zugegriffen: 02. Januar 2013).

Young, Justice and the Politics of Difference, Princeton/New Jersey 1990.

Zinsmeister, Mehrdimensionale Diskriminierung. Das Recht behinderter Frauen auf Gleichberechtigung und seine Gewährleistung durch Art. 3 GG und das einfache Recht, BadenBaden 2007. 


\title{
Inklusionsorientierung im Sozialraum - Verpflichtung und Herausforderung
}

\author{
Clemens Dannenbeck
}

I. Einführung - Der Inklusionsdiskurs

II. Feuilletonistische Randbemerkungen aus dem beschädigten Leben oder (fast) wie im richtigen Leben

1. Die Grenzen der Inklusion in der inklusiven Praxis 50

2. Ramponierte Teilhabe - der vermessene inklusive Sozialraum $\quad 50$

3. Einsicht in die eigene Verstricktheit 51

4. Inklusion einfach machen - und alles wird gut 51

III. Wissenschaftlicher Teil $\quad 51$

$\begin{array}{ll}\text { IV. Ausblick } & 56\end{array}$

\section{Einführung - Der Inklusionsdiskurs}

Im Folgenden wird versucht, die Notwendigkeit der stärkeren Berücksichtigung einer sozialräumlichen Perspektive in der politischen und öffentlichen Inklusionsdebatte zu begründen, um anschließend in Ansätzen herauszuarbeiten, zu welchen behindertenpolitischen Blickverschiebungen eine solche Fokussierung des Sozialraums führen würde. Ausgangspunkt der Argumentation ist folgende Wahrnehmung des Inklusionsdiskurses, wie er sich infolge der Ratifizierung der UN-BRK durch die Bundesrepublik Deutschland seit dem Jahr $2009^{1}$ präsentiert: Die Ratifizierung der UN-BRK führte zu einem (für manche durchaus überraschend) hohem Handlungsdruck und -bedarf auf diversen politischen Entscheidungsebenen, nicht zuletzt durch die mit der freiwilligen Ratifizierung des Fakultativprotokolls verbundenen inhärenten Kontroll- und Qualitätsmechanismen, die die Reaktionen und Umsetzungsprozesse national wie international beglei-

1 Die UN-Konvention über die Rechte von Menschen mit Behinderung ist seit dem 26. März 2009 in der Bundesrepublik Deutschland rechtsverbindlich. Grundlage ist das Gesetz zum Übereinkommen der Vereinten Nationen vom 13. Dezember 2006 über die Rechte von Menschen mit Behinderungen sowie das Fakultativprotokoll vom 13. Dezember 2006 zum Übereinkommen der Vereinten Nationen über die Rechte von Menschen mit Behinderungen (Deutsch, Englisch, Französisch); vgl. hierzu: http://www.institut-fuer-menschenrechte.de/de/monitoring-stelle.html (Stand 10. Juni 2012). 